\title{
Multipurpose silicon detector for (polarized) internal target experiments at the COSY Storage Ring in Jülich
}

\section{Anton Kononov ${ }^{1}$}

University of Ferrara and INFN, I-44100 Ferrara, Italy,

E-mail: anton.kononovestudent.unife.it

\begin{abstract}
A low-energy spin-physics program is being developed at the COSY storage ring. To support the planned experimental activities, different experimental tools for polarized beams and targets have been developed. The paper presents the physics case and the readiness of the setup for its realization.
\end{abstract}




\section{Introduction}

The PAX Collaboration is being involved in experiments employing polarized internal targets. In this framework, the team is since years exploring techniques to produce a beam of polarized antiprotons.

One objective of the investigations is the completion of the studies devoted to the identification of a viable method to produce an intense beam of polarized antiprotons undertaken some years ago by the PAX Collaboration[1]. After having successfully performed a spin-filtering test with protons using a transversely polarized hydrogen gas target [2], the PAX Collaboration plans to complete these studies with a test of the longitudinal polarization buildup at COSY. The outcome of these studies will be of utmost importance for the possible application of polarized antiprotons at the FAIR facility in Darmstadt.

A second physics goal consists of a time-invariance test accessing the null observable Ay,xz by scattering a vector polarized stored proton beam off a tensor polarized deuterium gas target[3]. The experimental environment at the COSY storage ring promises to improve the present upper limit on Time Reversal Violation obtained with a direct measurement by about two orders of magnitude.

To perform these investigations, the collaboration has developed a dedicated interaction point constituted a low- $\beta$ section, a storage cell, an atomic beam source and a vertex detector.

\section{Detector description}

The PAX silicon detector is high-acceptance, multipurpose silicon vertex detector installed around a storage cell where polarized or unpolarized H/D atoms and molecules can be injected. It has been designed to identify with proton- (anti)proton and proton-deuteron reactions in the energy range between and $30 \mathrm{MeV}$ and $200 \mathrm{MeV}$. For the case of polarized beam and/or target, the detector system, can serve as beam and

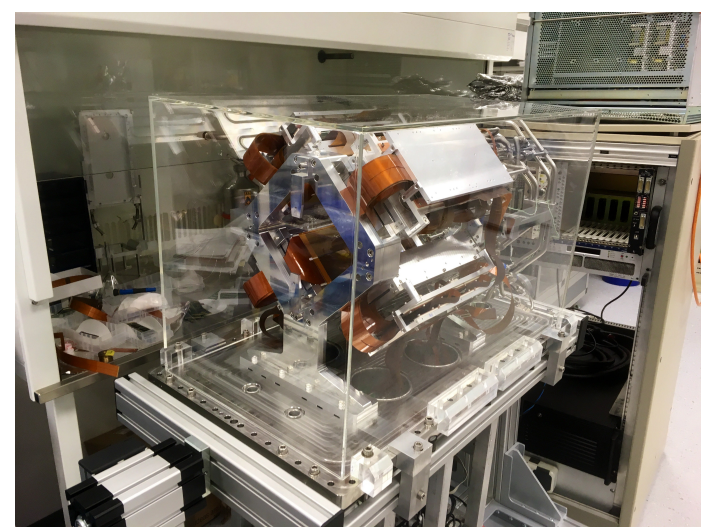

Fig. 1. The PAX multipurpose detector

target polarimeter by detection of asymmetries in proton-(anti)proton and proton 
deuteron-elastic scattering. The detector is composed of four identical quadrants combined in a diamond-shaped configuration around the storage cell (Fig. 1).

Each quadrant consists of three layers of double-sided silicon-strip sensors, mounted inside an aluminum box, and a front-end readout system. The sensors and the read-out electronics are cooled by separate circuits. The modularity allows for both for independent test of each quadrant with its associated electronics and for fast replacement in case of malfunctioning (Fig. 2).
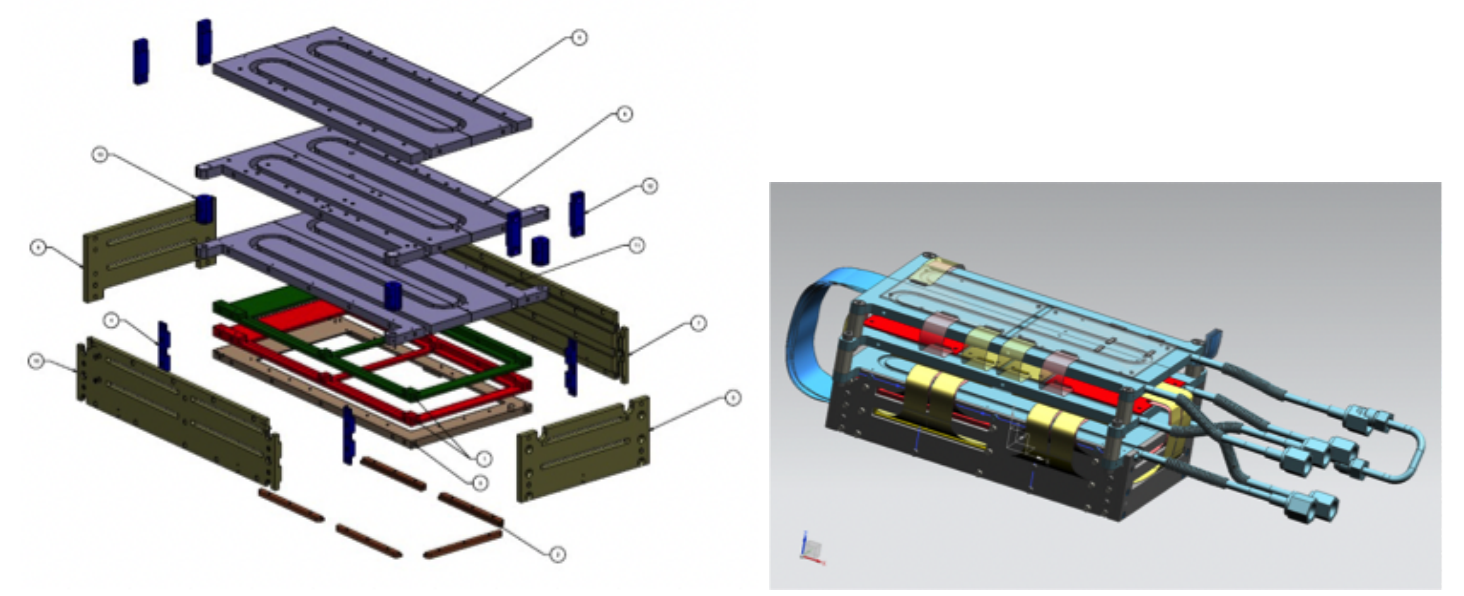

Fig. 2. Left: exploded view of a quadrant. In the center of the picture, the three frames of the individual detector layers (green, red and light brown) can be identified, above them the three cooling plates (violet) supporting the preamplifiers. Right: assembled quadrant.

The first layer, adapted from the recoil detector of the HERMES experiment [4], consists of two silicon sensors from the company MICRON Semiconductor [5], type TTT1, mounted side by side in a common ceramic frame. The detectors are $300 \mu \mathrm{m}$ thick and have an active area of $97.3 \mathrm{~mm} \times 97.3 \mathrm{~mm}$. Both contacts of the detectors are structured with 128 stripes (stripe width $702 \mu \mathrm{m}$, pitch $758 \mu \mathrm{m}$ ). Before assembly into the quadrant housing, the ceramic frame has been mounted on an aluminum frame and the strips have been ultrasonically bonded to the kapton foils connecting the detectors to the supply voltage and to a charge-sensitive preamplifier (Fig. 3-left).

The second layer have been realised with two silicon detectors from the company MICRON Semiconductor Ltd., but type TTT2. In this case, the sensors are individually mounted on the ceramic frame. The detectors are $300 \mu \mathrm{m}$ thick and the active area is $97.22 \mathrm{~mm} \times 97.22 \mathrm{~mm}$. Both contacts of the detectors are structured with 128 stripes (stripe width $700 \mu \mathrm{m}$, pitch $760 \mu \mathrm{m}$ ). The strips are bonded (bonded) to kapton films glued to the ceramic frames. The two detectors are then mounted side by side in an aluminum frame (Fig. 3-right). 

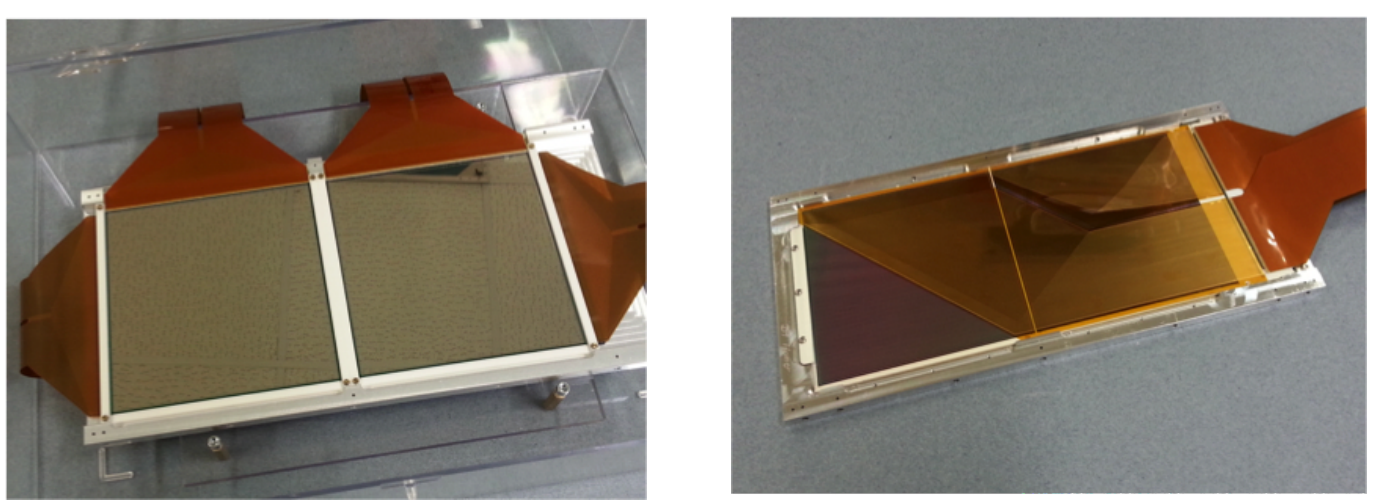

The

Fig. 3. Left: First layer of realised with the $300 \mu$ sensors recovered from the HERMES recoil detector; Right: second layer of the detector.

third layer consists of a single silicon sensor. It is also a TTT2 detector from MICRON Semiconductor Ltd., but with a thickness of $1000 \mu \mathrm{m}$. The third layer has been installed to increase to stopping power of the detector and extend the detector applicability to the identification of deuteron-breakup events.

\section{Openable storage cell}

The use of an openable storage cell is a highly desirable feature for an internal target experiment where a limitation of the ring acceptance can inhibit the intensity of the injected beam. The cell is opened at injection and closed after the beam has been cooled, and its emittance and size are reduced. The cell

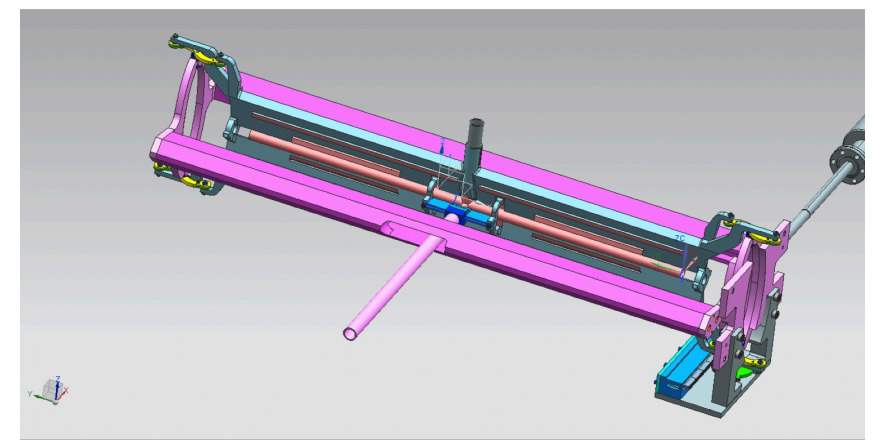

Fig.4. Storage cell in the closed position. The polarized gas in injected in the cell through a tube in the upper part of the cell (blue color in the picture), while a sample of the target gas diffuses to the diagnose system by through a side tube (pink color in the picture).

itself is $40 \mathrm{~cm}$ long and has an internal diameter in the closed position of $10 \mathrm{~mm}$. It has been realised with an Aluminum foil of 50 /mu thickness. Polarized gas can be injected in the cell from a tube installed in the upper part of the cell. A sample of the target gas diffuses from a side tube into the diagnose system (Fig. 4) 
In order to allow to operate on the cell without interference with the detector, a dedicated insertion mechanism has been realised. (Fig. 5).

To facilitate the assembly of the different quadrants and the insertion of the cell, in the detector system, a motor driven system that allows a $360^{\circ}$ rotation of the support flange has been realised.

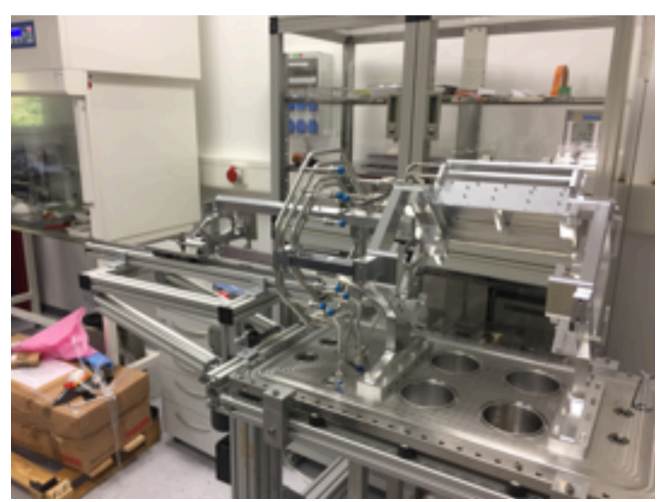

Fig. 5. Insertion mechanism of the cell in the detector. The system allows to insert and extract the cell without interference with the detector itself.

\section{Readout electronics}

Dedicated front-end boards for preamplification have been designed at FZJ and realised in Italy by ARTEL S.r.1 [6] (Fig. 6). In order to make partial use of the hardware and soft- ware already developed, the PCBs have been realised by using the VA32TA2.2 chips by Ideas [7] already adopted in the readout of the silicon telescopes of the ANKE experiment [8].

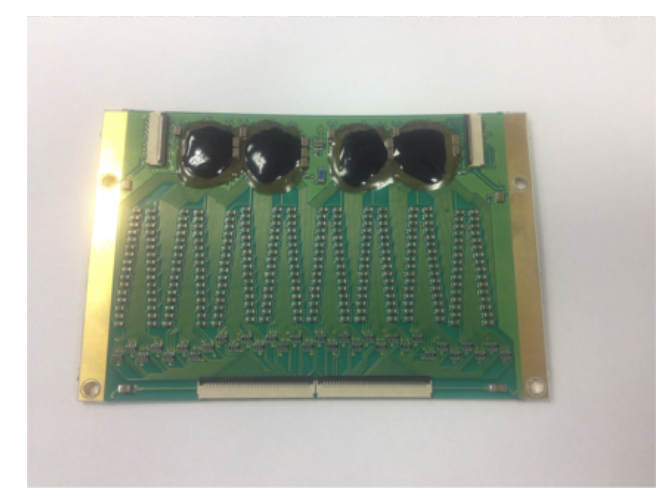

Fig. 6. Preamplifier board

\section{Commissioning}

After calibration of the single quadrants and sensors on an external test bench, the complete detector has been installed and commissioned in the COSY storage ring. The commissioning has been accomplished with an unpolarized proton beam at 135 $\mathrm{MeV}$ hitting vector polarized deuteron target. 
As described in sec. 2, each layer of the detector consisted of two stripped sensor sides,

one of them with the strips oriented along the beam axis and the other in the perpendicular direction. Thus, each layer measured a two- dimensional coordinate on a track. The average number of strips in a track hit was about two in the first two layers and four in the third thick layer, and a track coordinate was reconstructed as a weighted

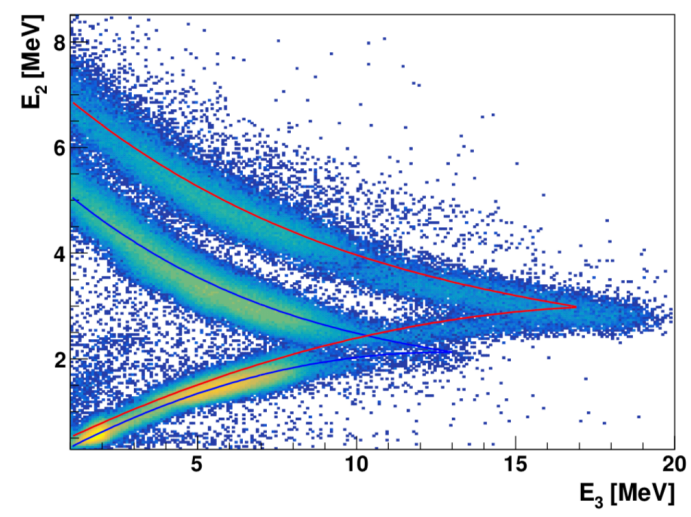

Fig. 7. Energy deposit in the second layer vs. one in the third for the tracks with $60^{\circ}<\theta<80^{\circ}$. The two upper bands contain deuterons and protons stopped in the third layer, with the deuteron band above. The lower rising band contains the particles passing through the third layer. The curves show position of the bands in the simulation: red for deuterons, blue for protons.

average according to the charge distribution in the strips fired. By extending the straight line of a track to the beam axis, one could find the longitudinal and transverse coordinates of the inter- action point with an accuracy (RMS) of about $2 \mathrm{~mm}$.

The deposited energy loss in the detector was calibrated with the use of a GEANT-based [9] simulation. In Fig. 7 a distribution is shown of the energy deposit in the second layer versus the one in the third. One can see loci of protons and deuterons that were stopped in the third layer and of those that passed it. To avoid thickening of the lines, this plot as obtained in a narrow track polar angular range around $70 \circ$. Analogous dependencies were observed

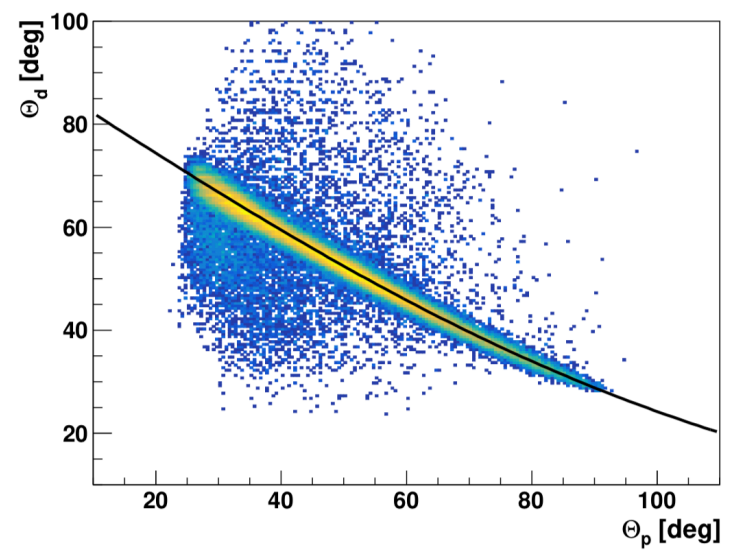

Fig. 8. Correlation of laboratory polar angles in two track events, selected by pdelastic azimuthal correlation. The energy loss cuts are applied to select protons and deuterons passing through the third layer. 
for the combination of the first and second layers. By comparing such plots from simulation with the correlations of the measured charges, one could obtain the energy calibration parameters. Fig. 7 demonstrates as well the possibility to identify the particles that are stopped in the second or third layer.

Fig. 8 shows the polar angle distribution of the identified pd-elastic events. The events were selected by requiring matching vertex coordinates for the two tracks and the

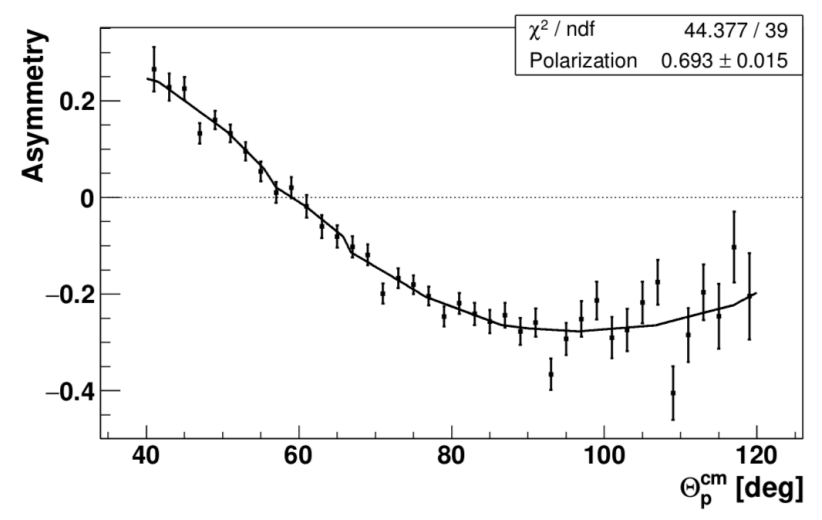

Fig. 9. Experimental asymmetry in pd-elastic scattering as a function of proton center-ofmass polar angle. The curve shows interpolated $\mathrm{A}_{\mathrm{y}}^{\mathrm{d}}$ data from Ref. [10] scaled to fit our data.

proper correlation of the azimuthal angles, the tracks were ordered using their energy loss. Both proton and deuteron were fast enough to penetrate the third layer when detected in coincidence. Despite the not ideal vacuum conditions in the target chamber during the beam time (no sensible dependence in the total detector rate could be detected with and without injection of polarized gas in the cell), the pd-elastic events could be cleanly selected with a background level of only $1.5 \%$.

In Fig. 9 the observed asymmetry $\varepsilon$ corrected for $\langle\cos \phi\rangle$ is presented for the pd-elastic reaction. The events were selected by the common vertex criterion, $p-d$ angular correlations and cuts on the energy loss.

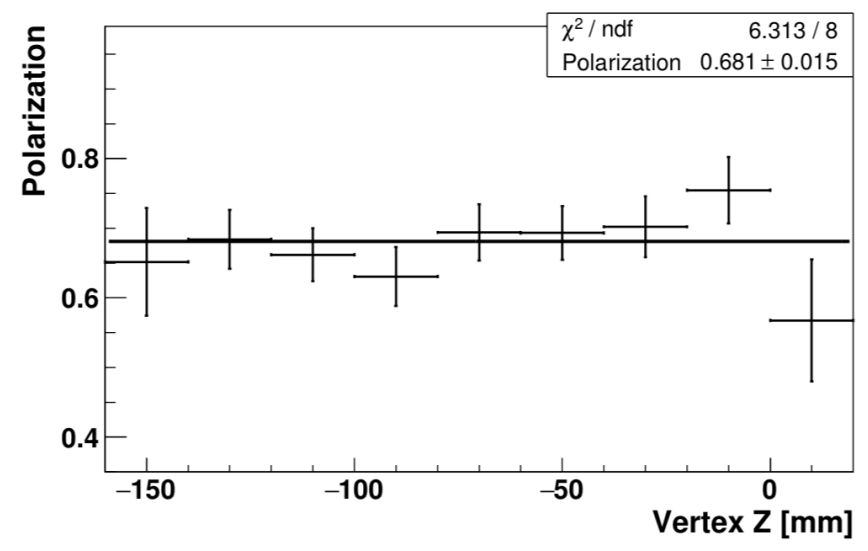

Fig. 10. Target polarization estimated as a function of the longitudinal vertex coordinate $\mathrm{Z}$ along the storage cell. The full line presents fit with a constant. 
Dependence of the polarization on longitudinal coordinate $\mathrm{Z}$ of the production point is shown in Fig. 10, where the origin corresponds to the cell and the detector center, and the axis is directed along the beam flow. The histogram values in each bin are obtained in the same way as demonstrated in Fig. 10. The range of $\mathrm{Z}$ shown is defined by the detector acceptance. One can see that no polarization loss is observed away from the injection tube of the cell located at $\mathrm{Z}=0$. This result could be obtained only from the measurement presented since the Breit-Rabi polarimeter only samples the gas from the center of the cell. 


\section{References}

1. Barone V, et al (2005) Antiproton-proton scattering experiments with polarization. hep-ex/0505054

2. Augustyniak W, et al (2012) Polarization of a stored beam by spin-filtering. Phys Lett B 718(1):64 69, DOI http://dx.doi.org/10.1016/j.physletb.2012. 10.030, URL http://www.sciencedirect.com/ science/article/pii/S0370269312010842

3. Valdau Y, Aksentyev A, Eversheim D, Lorentz B (2016) The physics program of PAX at COSY. J Phys Conf Ser 678(1):012,027, DOI 10.1088/1742-6596/ 678/1/012027

4. A. Borysienko et al., Nucl. Phys. B 125, (2003) 189.

5. Micron Semiconductor Ltd, 1 Royal Buildings, Lancing, West Sussex, BN15 8SJ, UK.

6. ARTEL S.r.1., Via Cassia 40, 52041 Civitella In Val Di Chi- ana (AR), Italy

7. https://ideas.no/

8. R. Schleichert, T. Krings, S. Merzliakov, A. Mussgiller, and D. Protic, IEEE Trans. Nucl. Sci. 50, 301 (2003).

9. S. Agostinelli et al., Nucl. Instum. Methods A 506, (2003) 250.

10.B. v. Przewoski et al., Phys. Rev. C 74, (2006) 064003. 